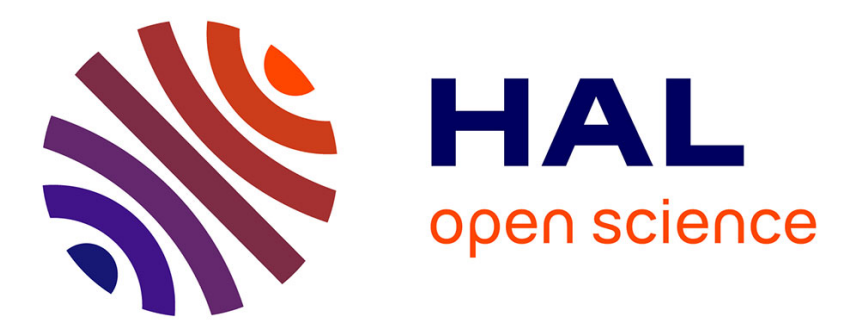

\title{
Etude théorique et expérimentale du couplage entre un concentrateur solaire et un récepteur haute température
}

\author{
G. Arnaud, G. Flamant, C. A. Muller, J.M. Badie, D. Hernandez
}

\section{To cite this version:}

G. Arnaud, G. Flamant, C. A. Muller, J.M. Badie, D. Hernandez. Etude théorique et expérimentale du couplage entre un concentrateur solaire et un récepteur haute température. Revue de Physique Appliquée, 1983, 18 (6), pp.327-334. 10.1051/rphysap:01983001806032700 . jpa-00245099

\section{HAL Id: jpa-00245099 https://hal.science/jpa-00245099}

Submitted on 1 Jan 1983

HAL is a multi-disciplinary open access archive for the deposit and dissemination of scientific research documents, whether they are published or not. The documents may come from teaching and research institutions in France or abroad, or from public or private research centers.
L'archive ouverte pluridisciplinaire HAL, est destinée au dépôt et à la diffusion de documents scientifiques de niveau recherche, publiés ou non, émanant des établissements d'enseignement et de recherche français ou étrangers, des laboratoires publics ou privés. 


\title{
Etude théorique et expérimentale du couplage entre un concentrateur solaire et un récepteur haute température $\left(^{+}\right)$
}

\author{
G. Arnaud, G. Flamant, C. Muller, J. M. Badie (*) et D. Hernandez (*) \\ Laboratoire d'Energétique Solaire, CNRS, BP 5, 66120 Odeillo, France \\ (Reçu le 24 janvier 1983, révisé le 28 février, accepté le 8 mars 1983)
}

\begin{abstract}
Résumé. - Une méthode de calcul de l'éclairement solaire en tout point, d'orientation quelconque, de la zone focale d'un concentrateur à double réflexion est développée. Ces données sont utilisées dans un programme de simulation numérique d'un récepteur haute température pour calculer la distribution de température de l'enceinte. Les résultats expérimentaux obtenus valident les approches théoriques. Une généralisation du modèle est envisageable dans le domaine des fours à rayonnement infra-rouge utilisant des émetteurs à réflecteurs paraboliques.
\end{abstract}

\begin{abstract}
A numerical method to calculate solar irradiation at any point of the focal zone of a double reflexion concentrator is proposed. The first part of the study concerned a paraboloidal perfect mirror and a faceted reflector. In the latter case, the optical errors due to bad orientation and manufacture defects are taken into account. Experimental solar flux density distribution in the focal zone of a $2 \mathrm{~kW}$ and of a $6 \mathrm{~kW}$ solar furnace are presented and compared with calculated data. Then the temperature profile along the wall of a high temperature receiver is computed when solar irradiation and wall heat transfer flux density are imposed. The comparison between experimental and theoretical data shows a good agreement when the receiver is a metallic tube equiped with thermocouples and fluxmeters. The model may be generalized to describe heat transfer inside industrial furnaces using parabolic I.R. lamps.
\end{abstract}

\section{Introduction.}

La conversion de l'énergie solaire à haute température ouvre des perspectives d'application importantes compte tenu de la quantité d'énergie thermique consommée à températures supérieures à $900^{\circ} \mathrm{C}$ : plus de $10 \%$ de la consommation totale d'énergie en France[1].

D'une part au plan thermodynamique, les rendements de conversion en électricité sont élevés entre 800 et $1200^{\circ} \mathrm{C}$ pour les systèmes utilisant des cycles à air [2]. D'autre part les industries grandes consommatrices d'énergie (métallurgie, liants hydrauliques...) mettent en ouvre des procédés de traitement thermochimique à haute température. Dans ces deux domaines la pénétration de l'énergie solaire est liée pour une part à la mise au point de récepteurs haute température destinés au chauffage de gaz $[3,4]$ et de réacteurs chimiques solaires gaz-solide [5-7].

Les contraintes thermiques liées aux températures de fonctionnement de ces composants se situent souvent à la limite supérieure de leur résistance mécanique. C'est pourquoi, il convient d'éviter toute « pointe de flux » pouvant entraîner une détérioration irréversible de l'absorbeur. Cet objectif peut être atteint par simulation numérique du concentrateur et du récepteur.

Pour un concentrateur donné, l'étude théorique doit permettre le calcul des profils de densité de flux totale et de température dans un récepteur de forme quelconque connaissant le flux d'échange aux parois.

Expérimentalement les résultats de la simulation numérique peuvent être vérifiés grâce à des maquettes chaudes équipées de thermocouples et de fluxmètres.

La présente étude développe l'ensemble de ces méthodes.

\section{Simulation numérique des concentrateurs solaires à double réflexion.}

Nous étudierons successivement le cas des paraboloïdes monoblocs et des concentrateurs à facettes. 2.1 PARABOLOİDES MONOBLOCS. - Les fours solaires de faible puissance $(<2 \mathrm{~kW})$ d'Odeillo sont des concentrateurs paraboliques monoblocs recevant un rayonnement parallèle réfléchi par un héliostat plan (Fig. 1a). Cette propriété (surface réfléchissante unique) permet de faire des hypothèses simplificatrices au

$\left(^{+}\right)$Demande de tirés à part à G. Flamant.

(*) Laboratoire des Uitra Réfractaires, CNRS, BP 5, 66120 Odeillo, France 

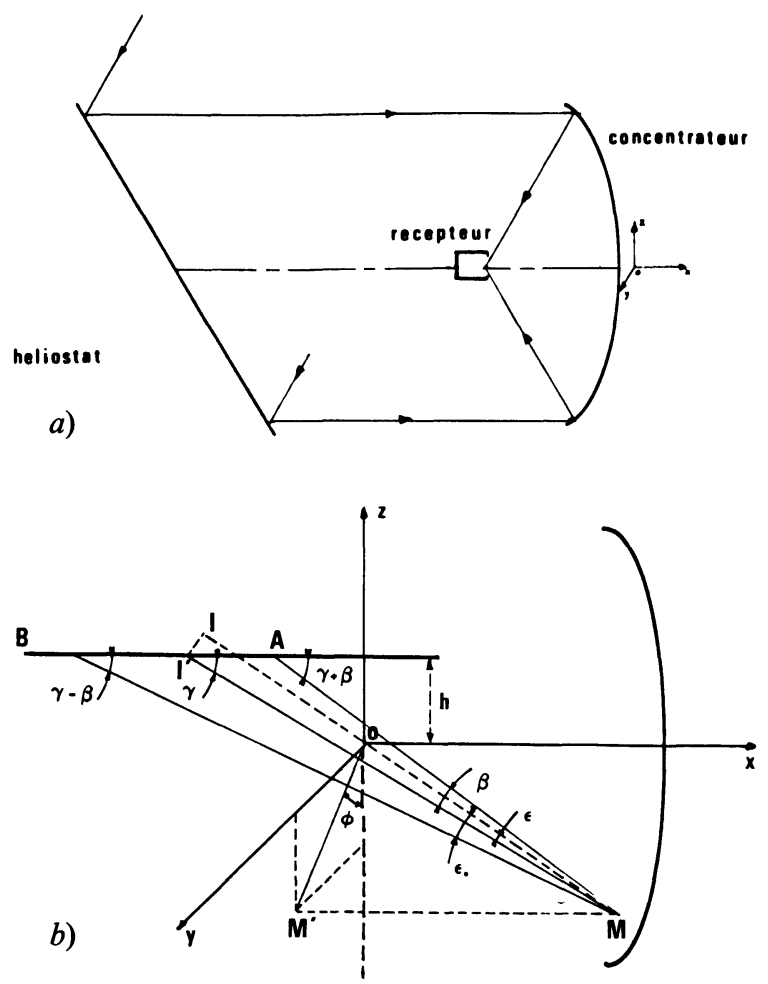

Fig. 1. - Concentrateur solaire de $2 \mathrm{~kW}, 1 a$ : Schéma d'ensemble. $1 b:$ Représentation géométrique utilisée pour le calcul de l'éclairement issu d'un point $\mathbf{M}$ du concentrateur.

[ $2 \mathrm{~kW}$ solar concentrator. $1 a:$ Scheme of the whole experimental set up. $1 b$ : Scheme used to calculate the solar irradiation from a point $\mathbf{M}$ located on the miror.] niveau du modèle, en particulier que la géométrie du système est parfaite. Les conséquences de cette hypothèse ont été développées par O. Kamada [8] :

- Premièrement, la distribution de la luminance dans le plan focal est la même que celle du disque solaire et peut être calculée par la fonction suivante :

$$
L_{\mathrm{s}}(\varepsilon)=L_{0} G(\varepsilon)=L_{0} \cos \left(m \varepsilon^{2}\right) .
$$

La valeur de $m$ est telle que $\cos \left(m \varepsilon_{0}^{2}\right)=0,55, \varepsilon_{0}=$ diamètre apparent du soleil $=16^{\prime}=0,00465$ rad., $L_{0}=$ luminance maximale au foyer ou au centre du soleil.

- Deuxièmement, le calcul de la distribution de l'éclairement sur une surface de révolution placée dans le volume focal peut être réalisé à l'aide de considérations géométriques simples.

Compte tenu de la forme de notre récepteur : cylindre de révolution dont l'axe est confondu avec l'axe focal ( $\mathrm{O} X$ sur la Fig. 1b), il suffit de déterminer le profil d'éclairement le long d'une génératrice pour obtenir la distribution sur toute la surface. Un faisceau conique de rayonnement solaire réfléchi au point $\mathbf{M}$ situé sur le concentrateur (voir Fig. 1) a pour image dans un plan tangentiel au cylindre une ellipse, intersection du cône d'axe MI et du plan. Ainsi le segment $\mathrm{AB}$, intersection de l'ellipse et de la génératrice, représente l'ensemble des points éclairés par $\mathbf{M}$. En chacun de ces points, l'éclairement est calculé par l'expression suivante [8]:

$$
\begin{aligned}
\mathrm{d}^{2} E\left(h^{\prime}, \theta, \phi\right)=\frac{\rho E_{0} m}{\pi \sin \left(m \varepsilon_{0}^{2}\right)} \sin ^{2} \theta \cos \phi \cos \left\{m\left(\frac{h}{2}\right)^{2}(1+\cos \theta)^{2} \times\right. \\
\left.\times\left[\left(\left(\frac{h^{\prime}}{h}\right) \sin \theta-\cos \theta \cos \phi\right)^{2}+\sin ^{2} \phi\right]\right\} \mathrm{d} \phi \mathrm{d} \theta .
\end{aligned}
$$

$\rho=$ coefficient de réflexion global (2 réflexions),

$E_{0}=$ éclairement solaire au sol,

$h=R / f \quad R=$ rayon du cylindre, $f=$ distance focale,

$h^{\prime}=x / f \quad x=$ abscisse du point $\mathrm{M}(x, y, z)$,

$\theta=$ angle $\mathrm{MOX} \quad 0<\theta<60^{\circ}$

$\phi=$ angle $\mathrm{M}^{\prime} \mathrm{OZ} Z^{\prime} \quad 0<\phi<360^{\circ}, \mathrm{M}^{\prime}$ est la projection de $\mathrm{M}$ dans le plan yoz.

Le profil d'éclairement global est calculé par intégration des flux élémentaires issus des points $\mathrm{M}, \boldsymbol{\theta}$ variant de 0 à $60^{\circ}, \phi$ de 0 à $360^{\circ}$.

\subsection{Concentrateur À FaCETtes. - Concentrateur} à axe vertical, le «four solaire de $6 \mathrm{~kW}$ » reçoit le rayonnement réfléchi par un héliostat plan de $27 \mathrm{~m}^{2}$ selon une direction parallèle à son axe de symétrie (cf. Fig. 2). Il est constitué de 306 miroirs hexagonaux sphériques de $13 \mathrm{~cm}$ de côté et $7,60 \mathrm{~m}$ de rayon de courbure disposés sur une calotte sphérique de $4 \mathrm{~m}$ de diamètre et de $3,80 \mathrm{~m}$ de rayon de courbure (distance égale à la distance focale).
Les limites physiques de la concentration du rayonnement solaire par ce type de dispositif sont liées à deux causes :

- Le soleil nous apparaît comme un disque de diamètre apparent égal à $2 \varepsilon_{0}$, dont la valeur moyenne est $32^{\prime}$.

- Les surfaces réfléchissantes ne sont pas parfaites (défauts de planéité ou de sphéricité) et présentent des imperfections d'orientations.

Ces écarts à l'idéalité provoquent une dispersion du rayonnement par rapport au cas idéal et donc une diminution de l'éclairement maximal au centre de la tache focale. 


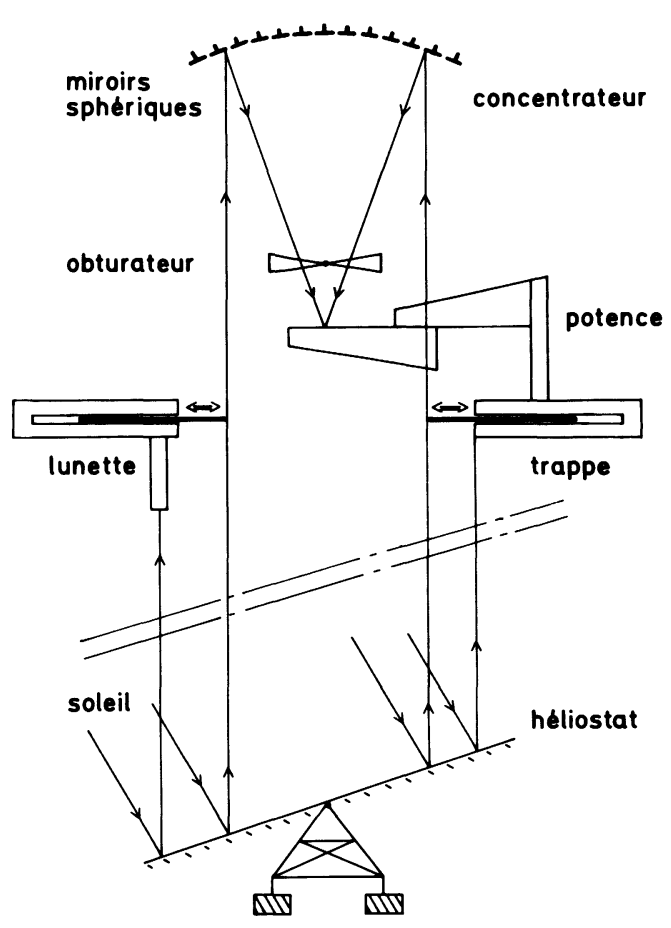

Fig. 2. - Concentrateur solaire de $6 \mathrm{~kW}$.

[Solar concentrator of $6 \mathrm{~kW}$.]

$\mathrm{Au}$ plan numérique nous avons traduit ces phénomènes physiques de la façon suivante : les écarts angulaires des normales aux miroirs par rapport à la direction correspondant à la perfection géométrique sont supposés suivre une loi de distribution gaussienne pour les deux surfaces réfléchissantes (héliostat plan et concentrateur). Les rayons réfléchis dans chaque cas suivent donc une loi de distribution gaussienne d'écart quadratique moyen double. La composition de deux distributions gaussiennes étant gaussienne, on peut donc considérer que les rayons correspondant à une direction donnée du soleil suivent, après la double réflexion, cette loi de distribution. On a donc construit un modèle (appelé ici "Soleil Fictif») qui ne se réfère plus aux chemins optiques suivis par les rayons solaires mais assimile la dispersion liée à la double réflexion à une dispersion au niveau des rayons issus du disque solaire (voir Fig. 3a).

Mathématiquement cela revient à faire le produit de convolution de la distribution réelle de l'énergie dans le disque solaire avec une distribution gaussienne de valeur moyenne nulle et dont l'écart quadratique moyen $\sigma$ est fonction des défauts de l'installation. $\mathrm{La}$ valeur de ce paramètre est déterminée en calant le modèle numérique sur les résultats expérimentaux.

La loi de distribution dans la direction $u(x, y)$ (voir Fig. 3) du disque solaire est égale à :

$$
\begin{aligned}
& f(x, y)=\frac{1}{\sqrt{2 \pi} \sigma} \exp \left[-\frac{x^{2}}{2 \sigma^{2}}\right] \cdot \frac{1}{\sqrt{2 \pi} \sigma} \\
& \times \exp \left[-\frac{y^{2}}{2 \sigma^{2}}\right] .
\end{aligned}
$$

La loi de variation de la luminance $L_{\mathrm{s}}$ du soleil en fonction de l'angle $u$ de la direction considérée avec la direction du centre théorique du soleil est calculée selon l'expression [9] :

$$
L_{\mathrm{s}}(x, y)=L_{\mathrm{s}}(u)=L_{0}\left(0,39+0,61 \sqrt{1-\frac{\sin ^{2} u}{\sin ^{2} \varepsilon_{0}}}\right)
$$

avec $L_{0}=1844,5 \mathrm{~W} \cdot \mathrm{cm}^{-2} \mathrm{st}^{-1}$.

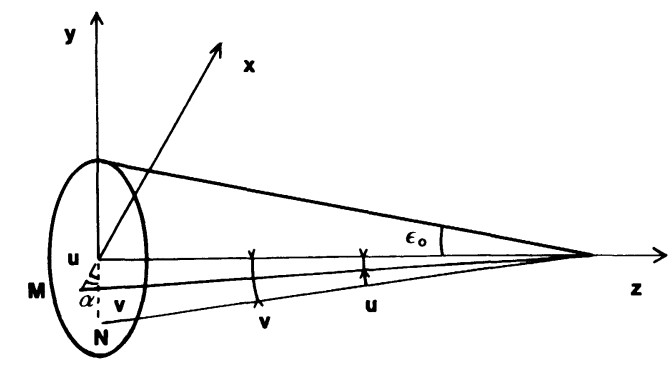

a

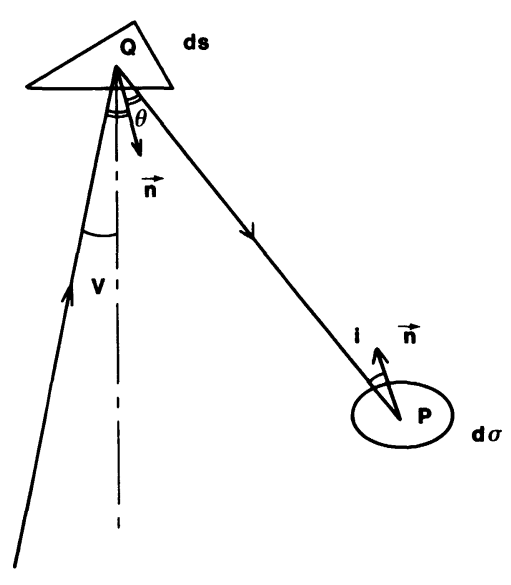

b

Fig. 3. - Représentation des éléments de calcul de l'éclairement pour le concentrateur de $6 \mathrm{~kW}$. $3 a$ : Repérage des angles dans le disque solaire. $3 b$ : Repérage des angles pour le calcul du flux arrivant sur l'élément de surface $\mathrm{d} \sigma$.

[Scheme of the various elements used for the calculation of solar irradiation from the $6 \mathrm{~kW}$ concentrator. $3 a$ : In the solar disc. $3 b$ : Calculation of solar irradiation from $\mathrm{d} s$ to $\mathrm{d} \sigma$.] 
La loi du soleil fictif dans la direction $v$, repérée par rapport au disque solaire sur la figure $1 a$ et par rapport aux facettes élémentaires $\mathrm{d} s$ du concentrateur sur la figure $1 b$, est donc calculée par :

$$
L\left(x_{1}, y_{1}\right)=L(v)=\int_{x} \int_{y} \frac{L_{\mathrm{s}}(x, y)}{2 \pi \sigma^{2}} \exp \left[-\frac{\left(x-x_{1}\right)^{2}}{2 \sigma^{2}}\right] \exp \left[-\frac{\left(y-y_{1}\right)^{2}}{2 \sigma^{2}}\right] \mathrm{d} x \mathrm{~d} y .
$$

Comme MN $=\left[\left(x-x_{1}\right)^{2}+\left(y-y_{1}\right)^{2}\right]^{1 / 2}=\left[u^{2}+v^{2}-2 u v \cos \alpha\right]^{1 / 2}$, l'expression précédente s'écrit :

$$
L(v)=\int_{\alpha=0}^{2 \pi} \int_{u=0}^{\varepsilon_{0}} \frac{1}{2 \pi \sigma^{2}} L_{\mathrm{s}}(u) \exp \left[-\frac{u^{2}+v^{2}-2 u v \cos \alpha}{2 \sigma^{2}}\right] u \mathrm{~d} u \mathrm{~d} \alpha .
$$

Pour être valable, ce calcul doit être effectué pour un grand nombre de surfaces élémentaires du concentrateur. Ainsi chacune des 306 facettes hexagonales est découpée en 96 éléments triangulaires de même surface $\mathrm{d} S$.

Soit $\mathrm{d} \sigma$ une surface élémentaire du cylindre placé au foyer, et $\mathrm{P}$ son centre, $\mathrm{Q}$ le centre de la facette élémentaire $\mathrm{d} S$. Le rayon incident faisant l'angle $V$ avec la verticale devient $\mathrm{QP}$ après réflexion (Fig. 3), le flux élémentaire $\mathrm{d} \phi$ arrivant sur $\mathrm{d} \sigma$ est calculé par l'expression

$$
\mathrm{d}^{2} \phi=L(v) \cdot \mathrm{d} s \cdot \cos i \frac{\mathrm{d} \sigma \cos \phi}{\mathrm{QP}^{2}} .
$$

$\theta=$ angle de $\mathrm{QP}$ avec la normale à $\mathrm{d} S$,

$i=$ angle de $\mathrm{QP}$ avec la normale à $\mathrm{d} \sigma$, par sommation sur les $306 \times 96$ éléments on obtient la densité de flux (éclairement) $\mathrm{d} \phi / \mathrm{d} \sigma$.

Dans un premier temps la valeur de l'écart quadratique moyen $\sigma$ a été déterminée par comparaison des mesures réalisées au foyer. Un très bon accord a été obtenu pour $\sigma=6,1[10]$.

\section{Modélisation des transferts par rayonnement dans le récepteur.}

La simulation numérique du concentrateur aboutit à la prévision de la distribution spatiale de l'éclairement solaire dans la zone focale. Ainsi l'objectif du « modèle récepteur " est le calcul de la distribution de température dans un récepteur solaire de forme quelconque connaissant la carte des éclairements solaires incidents et les flux d'échange aux parois. Inversement, le profil de température dans une cavité exposée au rayonnement concentré étant une donnée assez facilement accessible par l'expérience, il est intéressant de faire apparaître dans les équations du modèle, les relations liant les températures et les éclairements solaires directs pour calculer ces derniers à partir de la mesure du champ de température.

Les hypothèses de l'analyse théorique inspirée de Hottel et Poljak [11] sont les suivantes :

- Le récepteur est considéré comme une enceinte fermée dont l'ouverture est assimilée à une surface perpendiculaire à l'axe focal à zéro ${ }^{\circ} \mathrm{C}$ de facteur d'absorption égal à l'unité et de facteur de réflexion nul.

- Pour chaque surface composant l'enceinte, l'émission et la réflexion sont diffuses et isotropes.

- Les parois sont opaques et se comportent comme des corps gris dans les deux domaines spectraux visible et infra-rouge. Pour chaque domaine, on peut donc écrire :

et

$$
\varepsilon_{\mathrm{s}, \mathbf{k}}=\alpha_{\mathrm{s}, \mathbf{k}} \quad \varepsilon_{\mathrm{r}, \mathbf{k}}=\alpha_{\mathrm{s}, \mathbf{k}}
$$

$$
\varepsilon_{\mathrm{s}, \mathbf{k}}+\rho_{\mathrm{s}, \mathbf{k}}=1 \quad \varepsilon_{\mathrm{r}, \mathbf{k}}+\rho_{\mathrm{r}, \mathbf{k}}=1
$$

$\alpha=$ facteur total d'absorption, $\varepsilon=$ facteur total d'émission, $\rho=$ facteur total de réflexion, $\mathrm{s}=$ solaire, $\mathrm{r}=$ infra-rouge.

Enfin l'enceinte est décomposée en $N$ éléments isothermes, $\mathrm{k}$, dont la température est $T_{\mathrm{k}}$.

En négligeant les pertes par convection naturelle, le bilan thermique pour chaque élément peut s'écrire :

$$
q_{\mathrm{s}, \mathrm{k}}+q_{\mathrm{r}, \mathrm{k}}+q_{\mathrm{c}, \mathrm{k}}=0
$$

avec

$q_{\mathrm{s}, \mathrm{k}}=$ densité de flux solaire net (incident et réfléchi)

$q_{\mathrm{r}, \mathrm{k}}=$ densité de flux infra-rouge net

$q_{\mathrm{c}, \mathrm{k}}=$ densité de flux d'échange à travers la paroi (flux d'échange avec le caloporteur et/ou flux de perte).

L'écriture de l'égalité (1) pour les $N$ éléments conduit à $N$ équations à $3 N$ inconnues dont la résolution fait appel à des méthodes matricielles.

L'expression mathématique des divers éléments de l'égalité (1) fait l'objet des paragraphes suivants.

3. 1 Densité DE FluX SOlAIRE NET $q_{\mathrm{s}, \mathrm{k} \cdot}$ - - La distribution de l'éclairement solaire direct à l'intérieur d'un récepteur est généralement anisotrope. Nous noterons $E_{\mathrm{s}, \mathbf{k}}$ l'éclairement solaire direct arrivant sur l'élément $\mathrm{k}$. Soit $q_{\text {is,k }}$ la densité de flux solaire incident sur k (direct et réfléchis par les autres surfaces), $q_{\mathrm{es}, \mathrm{k}}$ la densité de flux solaire partant de $\mathrm{k}$ (radiosité), $F_{\mathrm{k}, \mathrm{j}}$ le facteur de forme entre la surface $k$ et la surface $j$.

$q_{\mathrm{is}, \mathrm{k}}=E_{\mathrm{s}, \mathrm{k}}+\sum_{\mathrm{j}=1}^{N} F_{\mathrm{k}, \mathrm{j}} q_{\mathrm{es}, \mathrm{j}}$

$q_{\mathrm{es}, \mathrm{k}}=\left(1-\varepsilon_{\mathrm{s}, \mathrm{k}}\right) q_{\mathrm{is}, \mathrm{k}}$

$q_{\mathrm{s}, \mathrm{k}}=q_{\mathrm{is}, \mathrm{k}}-q_{\mathrm{es}, \mathrm{k}}=\frac{\varepsilon_{\mathrm{s}, \mathrm{k}}}{\left(1-\varepsilon_{\mathrm{s}, \mathrm{k}}\right)} q_{\mathrm{es}, \mathrm{k}}=\varepsilon_{\mathrm{s}, \mathrm{k}} q_{\mathrm{is}, \mathrm{k}}$. 
Les équations (2) et (3) permettent aussi de lier la densité de flux solaire incident et l'éclairement direct

$$
E_{\mathrm{s}, \mathrm{k}}=\sum_{\mathrm{j}=1}^{N}\left[\delta_{\mathrm{k}, \mathrm{j}}-F_{\mathrm{k}, \mathrm{j}}\left(1-\varepsilon_{\mathrm{s}, \mathrm{j}}\right)\right] q_{\mathrm{is}, \mathrm{j}} .
$$

$\delta_{\mathbf{k}, \mathbf{j}}=$ symbole de Kroneker.

Le système d'équation permet de déterminer la densité de flux solaire net connaissant la distribution d'éclairement solaire direct à partir du système matriciel (5) et de l'équation (4).

3.2 Densité De FluX INFRA-ROUGe NET $q_{\mathrm{r}, \mathrm{k} \cdot}-$ La densité de flux infra-rouge net pour un élément $\mathbf{k}$ peut être positive ou négative selon les écarts relatifs de température entre les éléments. En utilisant le même symbolisme que précédemment nous avons :

$$
\begin{aligned}
q_{\mathrm{ir}, \mathrm{k}} & =\sum_{\mathrm{j}=1}^{N} F_{\mathrm{k}, \mathrm{j}} q_{\mathrm{er}, \mathrm{j}}, \\
q_{\mathrm{er}, \mathrm{k}} & =\varepsilon_{\mathrm{r}, \mathrm{k}} \sigma T_{\mathrm{k}}^{4}+\left(1-\varepsilon_{\mathrm{r}, \mathrm{k}}\right) q_{\mathrm{ir}, \mathrm{k}}, \\
q_{\mathrm{r}, \mathrm{k}} & =q_{\mathrm{ir}, \mathbf{k}}-q_{\mathrm{er}, \mathbf{k}}=\sum_{\mathrm{j}=1}^{N}\left(F_{\mathrm{k}, \mathrm{j}}-\delta_{\mathrm{k}, \mathrm{j}}\right) q_{\mathrm{er}, \mathrm{j}} .
\end{aligned}
$$

Les deux écritures suivantes peuvent être aussi utilisées :

$$
A_{\mathrm{k}} q_{\mathrm{r}, \mathrm{k}}=\sum_{\mathrm{j}=1}^{N} \sigma \mathcal{F}_{\mathrm{k}, \mathrm{j}}\left(T_{\mathrm{j}}^{4}-T_{\mathrm{k}}^{4}\right),
$$

$A_{\mathrm{k}}=$ aire de la surface $\mathrm{k}$, avec

$$
\begin{aligned}
\mathcal{F}_{\mathrm{k}, \mathrm{j}}=1 /\left[\left(1-\varepsilon_{\mathrm{k}}\right) / A_{\mathrm{k}} \varepsilon_{\mathrm{k}}+1 / A_{\mathrm{k}} F_{\mathrm{k}, \mathrm{j}}\right. & + \\
+ & \left.\left(1-\varepsilon_{\mathrm{j}}\right) / A_{\mathrm{j}} \varepsilon_{\mathrm{j}}\right] . \\
\sum_{\mathrm{j}=1}^{N}\left[\frac{\delta_{\mathrm{k}, \mathrm{j}}}{\varepsilon_{\mathrm{r}, \mathrm{j}}}-F_{\mathrm{kj}} \frac{\left(1-\varepsilon_{\mathrm{r}, \mathrm{j}}\right)}{\varepsilon_{\mathrm{r}, \mathrm{j}}}\right] q_{\mathrm{r}, \mathrm{k}}=\sum_{\mathrm{j}=1}^{N}( & \left.\delta_{\mathrm{k}, \mathrm{j}}-F_{\mathrm{k}, \mathrm{j}}\right) \times \\
& \times \sigma T_{\mathrm{j}}^{4} . \quad(10)
\end{aligned}
$$

Le flux $q_{\mathrm{r}, \mathrm{k}}$ peut être déterminé à partir de la mesure des températures grâce aux équations (10) ou (9).

3. 3 DeNSITÉ DE FLUX DE TRANSFERT À LA PAROI $q_{\mathrm{c}, \mathrm{k} \cdot \mathrm{-}}$ Deux types de flux, conductif et convectif, peuvent etre pris en compte : entre éléments constituant le récepteur (flux axial) et à travers chaque élément vers le caloporteur et/ou l'isolant (flux radial). Selon les cas, le flux de conduction entre éléments sera pris ou non en considération.

L'expression classique (11) permet la détermination de $q_{\mathrm{c}, \mathrm{k}}$ à partir de la mesure des températures et des propriétés thermiques du fluide de transfert

$$
q_{\mathrm{c}, \mathbf{k}}=K\left(T_{\mathbf{k}}-T_{\mathbf{k}}^{*}\right) .
$$

$T_{\mathrm{k}}^{*}=$ température moyenne de la paroi, côté caloporteur.

En conclusion l'ensemble des équations précédentes montrent que, connaissant la carte des flux solaires directs et des températures, on peut calculer la distri- bution de densité de flux globale dans la cavité; ou que partant du profil d'éclairement solaire dans un récepteur, il existe une relation biunivoque liant la température de l'absorbeur et le flux d'échange à la paroi délimitant le domaine d'utilisation du système.

\section{Dispositif expérimental.}

4.1 Concentrateurs. - Les maquettes ont été testées à l'aide des deux types de concentrateurs solaires présentés au premier chapitre. En ce qui concerne le miroir paraboloïde de $2 \mathrm{~kW}$ à axe horizontal, la puissance est modulée grâce à des écrans mobiles placés entre l'héliostat plan et le concentrateur. Dans le cas du concentrateur sphérique de $6 \mathrm{~kW}$ cette fonction est remplie par un obturateur à pales radiales placé entre le plan focal et la calotte sphérique réfléchissante (voir Fig. 2).

4. 2 RÉCEPTEUR. - Nous avons choisi des tubes en acier réfractaire $(25 \% \mathrm{Ni}, 20 \% \mathrm{Cr})$ pour diverses raisons :

- La résistance à l'oxydation jusqu'à $1150^{\circ} \mathrm{C}$.

- La géométrie simple, permettant de valider les modèles à l'aide d'enceintes pour lesquelles les facteurs de forme sont connus.

- Les applications potentielles en génie chimique solaire : four rotatif, lit fluidisé annulaire [12].

Les caractéristiques géométriques des tubes et les propriétés radiatives des surfaces utilisées pour la modélisation sont réunies dans le tableau I.

Tableau I.

$\begin{array}{lll}\text { Concentrateur } & 2 \mathrm{~kW} & 6 \mathrm{~kW} \\ \text { Diamètre }(\mathrm{m}) & 0,052 & 0,095 \\ \text { Longueur }(\mathrm{m}) & 0,12 & 0,20 \\ \text { Epaisseur }(\mathrm{m}) & 0,002 & 0,0045 \\ \varepsilon_{\mathrm{s}}, \varepsilon_{\mathrm{r}} & 0,7 ; 0,8 & 0,7 ; 0,8\end{array}$

Les tubes sont isolés thermiquement par $5 \mathrm{~cm}$ de laine, silico-alumineuse. Un diaphragme placé dans le plan focal limite l'ouverture d'entrée du rayonnement.

4.3 Mesures. - Deux types de mesures ont été effectuées : températures et flux.

Une rampe de thermocouples chromel-alumel reliée à une centrale d'acquisition de données est disposée le long d'une génératrice du cylindre en vue de la détermination du profil axial de température. Les soudures des couples thermoélectriques sont positionnées dans des logements usinés dans la paroi et maintenus en contact de la surface à $1 \mathrm{~mm}$ de la paroi interne par contrainte mécanique (ressorts de rappel).

La distribution de l'éclairement est mesurée à l'aide d'un fluxmètre à gradient radial type "Gardon" préalablement étalonné par calorimétrie.

La mesure simultanée des champs de température et de la densité de flux total a été réalisée en disposant un fluxmètre mobile le long d'une fenêtre usinée selon une génératrice du cylindre diamétralement opposée à la rampe de thermocouples. 
L'ensemble du dispositif expérimental est représenté sur la figure 4.

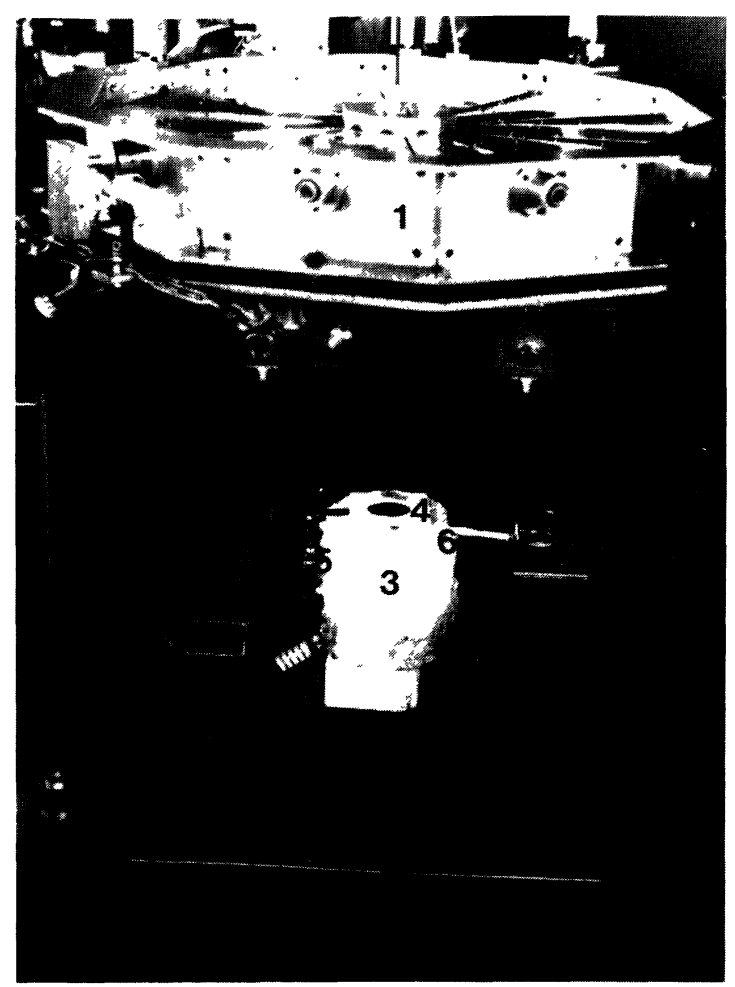

Fig. 4. - Dispositif expérimental placé au foyer du concentrateur de $6 \mathrm{~kW} .1$ : Obturateur (shutter). 2 : Potence (support). 3 : Tube récepteur (receiver). 4 : Diaphragme (aperture). 5 : Thermocouples. 6 : Fluxmètres (fluxmeters).

[Experimental set up located at the focus of the $6 \mathrm{~kW}$ solar concentrator.]

\section{Résultats.}

Les différentes mesures expérimentales réalisées concernent :

1) La distribution axiale de l'éclairement direct le long d'une génératrice.

2) Le profil axial de température dans le récepteur.

3) Les distributions axiales de température et de densité de flux total (solaire et IR).

Les mesures de type 1 permettent de valider le «modèle concentrateur» tandis que les mesures du type 2 et 3 sont destinées à vérifier le « modèle récepteur $"$.

5. 1 Concentrateur De $2 \mathrm{~kW}$. - Sur la figure 5 sont comparés les éclairements théorique et expérimental le long d'une génératrice d'un cylindre de $5,25 \mathrm{~cm}$ de diamètre disposée parallèlement à l'axe focal. On remarque que l'approche théorique simplifiée du modèle $2 \mathrm{~kW}$ est suffisante pour décrire la réalité.

La figure 6 montre les distributions de densité de flux et de température dans le récepteur tubulaire. Le profil température (courbe 3 ) est mesuré, les profils de densité de flux sont calculés. La courbe 1

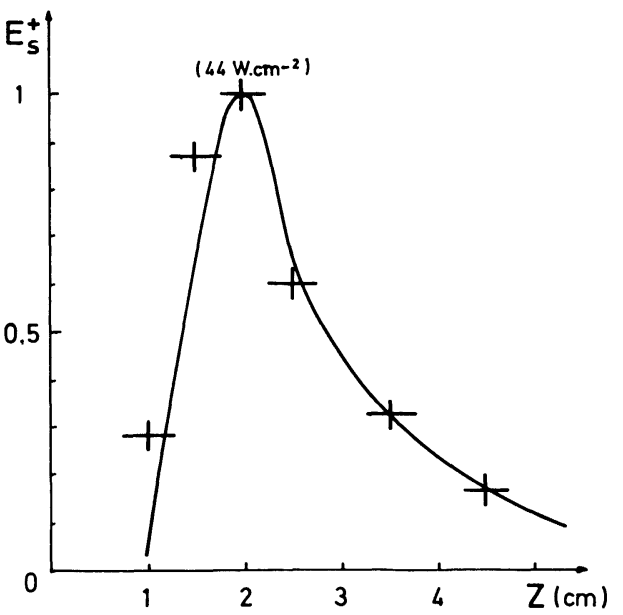

Fig. 5. - Eclairement solaire théorique (trait continu) et expérimental (croix) le long d'une génératrice située à $2,6 \mathrm{~cm}$ de l'axe focal du concentrateur de $2 \mathrm{~kW} . E_{\mathrm{s}}^{+}=$ $E_{\mathrm{s} \text { max }} / E_{\mathrm{s}}$

[Theoretical (continuous-line) and experimental (points) solar irradiation along a line located at $2.6 \mathrm{~cm}$ of the focal axis.]

correspond à l'éclairement solaire direct tandis que la courbe 2 représente la densité de flux solaire incident global (incident plus réfléchi) déduit du profil de température (courbe 3). Cette dernière courbe montre l'importance relative des réflexions multiples du rayonnement solaire direct sur la redistribution interne du flux.

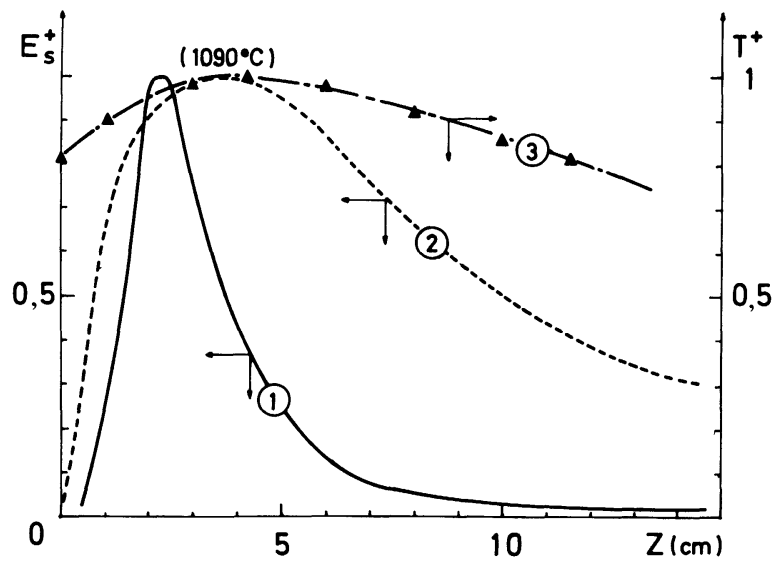

Fig. 6. - Profils de densité de flux $(1,2)$ et de température (3) dans un tube métallique de 5,2 $\mathrm{cm}$ de diamètre placé au foyer du "concentrateur de $2 \mathrm{~kW}$ »-puissance utile : $750 \mathrm{~W}, E_{\mathrm{s}}^{+}=E_{\mathrm{s} \max } / E_{\mathrm{s}} .1:$ Eclairement solaire direct (solar irradiation). 2 : Densité de flux solaire totale : direct + réfléchi (total solar irradiation : incident + reflected). 3 : Température de paroi (wall temperature).

[Solar irradiation $(1,2)$ and temperature (3) distribution along a metallic tube of $5.2 \mathrm{~cm}$ in diameter located at the focus of the « $2 \mathrm{~kW}$ solar furnace » useful power : $750 \mathrm{~W}$.]

5.2 Concentrateur de $6 \mathrm{~kW}$. - Les profils de densité de flux solaire direct le long d'une génératrice située à $4,75 \mathrm{~cm}$ de l'axe focal sont réunis sur la figure 7 . 


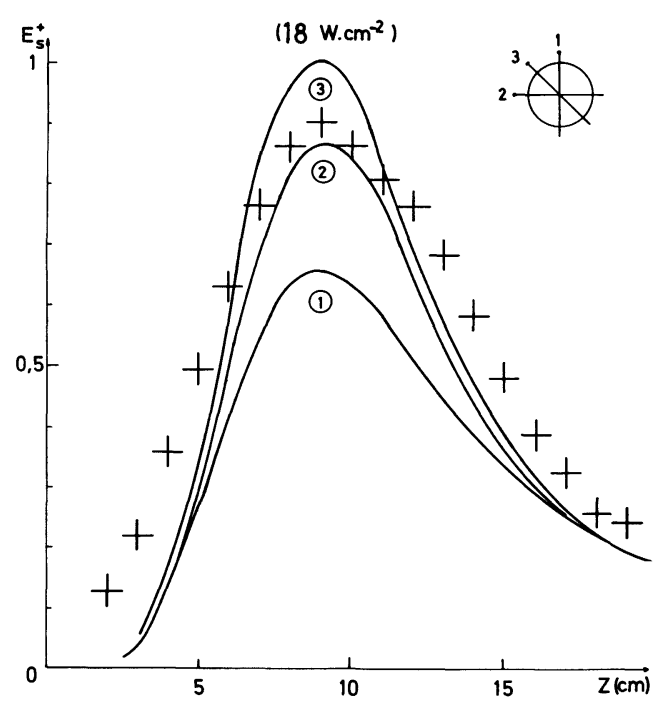

Fig. 7. - Distributions de l'éclairement solaire théorique (courbes 1, 2,3) et expérimental (points) le long d'une génératrice placée à $4,75 \mathrm{~cm}$ de l'axe focal du concentrateur de $6 \mathrm{~kW} . E_{\mathrm{s}}^{+}=E_{\mathrm{s} \max } / E_{\mathrm{s}} .1$ : Axe Nord-Sud. 2 : Axe OuestEst (axe des mesures). 3 : Axe Nord-Ouest-Sud-Est.

[Distributions of the solar theoretical irradiation (curves $\left.\mathrm{n}^{\mathrm{os}} 1,2,3\right)$ and experimental : points along a line located at $4.75 \mathrm{~cm}$ of the focal axis of the $6 \mathrm{~kW}$ concentrator.]

La comparaison des distributions théoriques (courbe 2) et expérimentales indique un étalement plus prononcé pour cette dernière mais l'écart moyen est de l'ordre de $20 \%$. Cet écart peut être attribué à l'imprécision de la simulation numérique dans les régions éloignées de la zone focale et aux erreurs de positionnement de la sonde de mesure. Les courbes 1, 2 et 3 correspondant aux axes Nord-Sud, Est-Ouest et Nord-Ouest, SudEst respectivement mettent en évidence la dissymétrie de l'éclairement direct dans des plans perpendiculaires au plan focal. Cette différence est liée, pour l'essentiel, à la présence de la potence support du récepteur solaire interposée entre le rayonnement solaire réfléchi par l'héliostat et le concentrateur.

Les courbes de la figure 8 illustrent la démarche générale de notre étude. Le tracé numéroté 1 correspond au profil de température calculé (modèle récepteur) à partir de la distribution d'éclairement solaire direct obtenue par le "modèle concentrateur». Le niveau de température de la zone quasi isotherme prévu par la simulation numérique est proche de la réalité. La valeur du gradient moyen de température dans la zone d'entrée du tube est comprise entre 40 et $50^{\circ} \mathrm{C} / \mathrm{cm}$ dans les deux cas. Par contre on observe un décalage du profil thermique vers le fond du tube. Cet écart est lié à la présence du diaphragme d'entrée dont le rôle n'est pas bien pris en compte dans la simulation numérique. Une meilleure approche est symbolisée par la courbe $\mathrm{n}^{\mathrm{o}} 2$ qui tient compte de l'effet de la conduction axiale entre les éléments du cylindre (découpé en 14 couronnes). La distribution $\mathrm{n}^{\mathrm{o}} 3$ est le résultat du calcul réalisé grâce au pro-

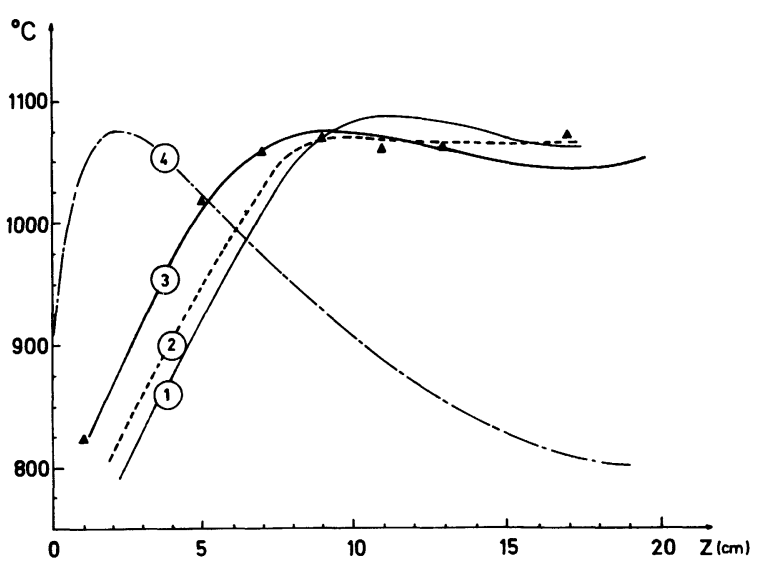

Fig. 8. - Profils de température théorique (courbes $1,2,3,4)$ et expérimental (triangle) dans un cylindre de $9,5 \mathrm{~cm}$ de diamètre placé au foyer du " concentrateur de $6 \mathrm{~kW}$ » puissance utile : 1600 W. 1 : Profil de température calculé à partir de la distribution de l'éclairement théorique (computed profile using theoretical solar irradiation). 2 : Même que 1 en introduisant le transfert conductif $(1+$ conductive flux $)$. 3 : Profil de température calculé à partir de la distribution expérimentale de l'éclairement solaire (computed profile using expérimental solar irradiation). 4 : Profil de température calculé en faisant l'hypothèse d'une distribution isotrope du rayonnement solaire à l'entrée du tube. (Computed profile assuming an isotrope distribution of solar irradiation on the aperture.)

[Theoretical temperature profiles (curves 1, 2, 3, 4) and experimental one (points) inside a cylinder of $9.5 \mathrm{~cm}$ in diameter located at the focus of the " $6 \mathrm{~kW}$ concentrator » useful power $1600 \mathrm{~W}$.]

gramme récepteur avec la distribution d'éclairement solaire direct mesurée expérimentalement. On note le bon accord entre le profil mesuré (triangles) et

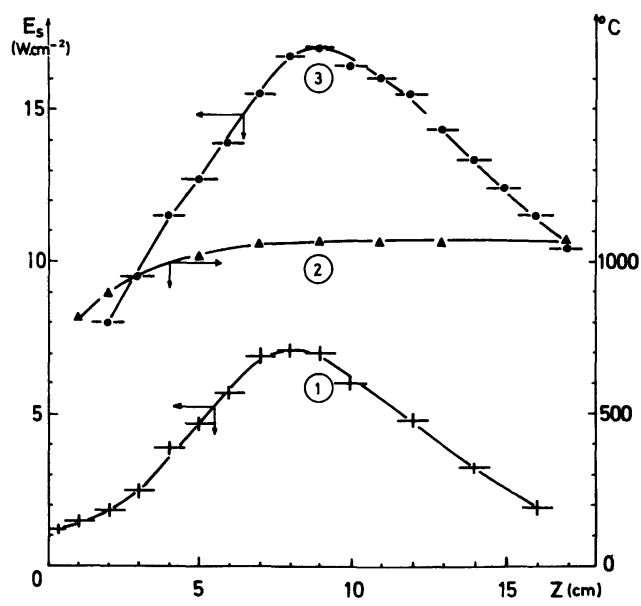

Fig. 9. - Distributions de densité de flux et de température expérimentale dans un cylindre de $9,5 \mathrm{~cm}$ de diamètre placé au foyer du "concentrateur de $6 \mathrm{~kW}$ » puissance utile $1600 \mathrm{~W} . E_{\mathrm{s}}^{+}=E_{\mathrm{s} \max } / E_{\mathrm{s}} .1$ : Eclairement solaire direct (solar irradiation). 2 : Température de paroi (wall temperature). 3 : Densité de flux totale (total flux density).

[Flux density and temperature distributions inside a cylinder of $9.5 \mathrm{~cm}$ in diameter located at the focus of « the $6 \mathrm{~kW}$ concentrator » useful power $1600 \mathrm{~W}$.] 
calculé (trait continu). Le tracé no 4 représente la distribution de température obtenue en faisant l'hypothèse d'une distribution isotrope de l'éclairement direct sur l'ouverture d'entrée.

Enfin la figure 9 représente les profils expérimentaux d'éclairement solaire direct (courbe 1), de température (courbe 2) et de densité de flux total (courbe 3). La comparaison des profils 1 et 3 montre l'importance du flux infra-rouge dans la cavité.

\section{Conclusion.}

Les simulations numériques développées concernant les concentrateurs et les récepteurs haute température conduisent à des distributions de densité de flux et de températures en bon accord avec les mesures expéri- mentales. Au plan pratique, ces outils doivent permettre, d'une part la conception de récepteurs solaires à géométrie variée selon le niveau et la distribution de température souhaitée, compte tenu des flux d'échange aux parois escomptés. D'autre part le modèle peut être étendu à certains dispositifs de chauffage industriel par rayonnement infra-rouge (à des températures inférieures à $250^{\circ} \mathrm{C}$ ) utilisant des émetteurs à réflecteurs paraboliques. En effet, le rayonnement issu de ces dispositifs peut être décrit par une distribution gaussienne. Chaque lampe est alors équivalente à une facette élémentaire de la simulation numérique décrite dans cet article. Cette généralisation prend de l'importance dans le contexte d'un accroissement de l'utilisation de l'électricité dans l'industrie du séchage par exemple.

\section{Bibliographie}

[1] Deflandre, J., Moisan, F., Matarasso, P., Valette, F., « Les applications de la chaleur solaire dans l'agriculture et l'industrie ». Entropie 96 (1981) 104-127.

[2] Peube, J. L., Blay, D., « Systèmes de conversion thermodynamique à haute température appliquées à l'énergie solaire ». Rev. Int. Hautes Temp. Réfract. 15 (1978) 93-98.

[3] GintZ, J., Bartlett, D., Zentmer, R., « 1 MWt bench model solar receiver test program ". Revue Phys. Appl. 15 (1980) 263-368.

[4] Wintrebert, G., Blay, D., Ortiz, F., Etude d'un récepteur solaire à haute température pour cycle thermodynamique à gaz. Système solaire thermodynamique 15-20 juin, Marseille (Ed. du CNRS) 1980.

[5] Flamant, G., «Thermochimie solaire à hautes températures. Résultats expérimentaux. Quelques perspectives d'application ". Revue Phys. Appl. 15 (1980) 503-511.

[6] Olalde, G., Peube, J. L., Daguenet, M., « Theoretical study of gas heated in a porous material subjected to a concentrated solar radiation ». Revue Phys. Appl. 15 (1980) 423-426.

[7] Royère, C., "Les applications du four solaire de $1000 \mathrm{~kW}$ du CNRS à ODEILLO ». Entropie 97 (1981) 147-160.

[8] KAMADA, O., "Theoretical concentration and attainable temperature in solar furnaces ». Solar Energy 9, no 1 (1965) 39-47.

[9] Le Phat Vinh, A., Trombe, E., « Etudes théoriques sur la concentration du rayonnement solaire et procédé de mesure ". Cah. AFEDES no 3, nov. (1971)

[10] Arnaud, G., Flamant, G., Olalde, G., Robert, J. F., "Les fours solaires de recherche du Laboratoire d'énergétique solaire d'ODEILLO ». Entropie 97 (1981) 135-146.

[11] Hottel, PolJak, dans Thermal radiation heat transfer Siegel and Howell (McGraw Hill Company) 1972.

[12] Badie, J. M., Bonet, C., Faure, M., Flamant, G. Foro, R., Hernandez, D., « Decarbonation of calcite and phosphate rock in solar chemical reactors ». Chem. Eng. Sci. 35 (1980) 413. 